PROCEEDINGS OF THE

AMERICAN MATHEMATICAL SOCIETY

Volume 135, Number 10, October 2007, Pages 3181-3185

S 0002-9939(07)08826-0

Article electronically published on May 10, 2007

\title{
SVEP FOR MULTIPLIERS ON A FAITHFUL COMMUTATIVE BANACH ALGEBRA
}

\author{
JANKO BRAČIČ AND MARTIN JESENKO
}

(Communicated by Joseph A. Ball)

\begin{abstract}
We give some sufficient conditions that each multiplier on a faithful commutative Banach algebra has SVEP. On the other hand, we show that there exist a faithful commutative Banach algebra and a multiplier on it without SVEP. Such examples of multipliers can actually be found within the class of multiplication operators on unital commutative Banach algebras. This answers in negative a question that is stated as Open problem 6.2.1 by Laursen and Neumann, 2000.
\end{abstract}

\section{INTRODUCTION}

A mapping $T: \mathcal{A} \rightarrow \mathcal{A}$ on a commutative complex Banach algebra $\mathcal{A}$ is a multiplier if

$$
a(T b)=(T a) b \quad \text { for all } \quad a, b \in \mathcal{A} .
$$

For every element $a \in \mathcal{A}$, the multiplication operator $L_{a}: \mathcal{A} \rightarrow \mathcal{A}$ that is given by $L_{a} b=a b(b \in \mathcal{A})$ is clearly a multiplier. When $\mathcal{A}$ has a unit, say $e$, the converse holds. Namely, if $T$ is a multiplier on $\mathcal{A}$, then $T b=e(T b)=(T e) b$, for all $b \in \mathcal{A}$, which means that $T$ is a multiplication operator induced by $T e$ on $\mathcal{A}$.

Let $L(\mathcal{A})$ be the set of all multiplication operators on $\mathcal{A}$. For a non-unital commutative Banach algebra $\mathcal{A}$, the set $M(\mathcal{A})$ of all multipliers on $\mathcal{A}$ may be larger than $L(\mathcal{A})$. However, if $\mathcal{A}$ is faithful in the sense that, for $a \in \mathcal{A}$, the products $a b$ $(b \in \mathcal{A})$ are all zero if and only if $a=0$, then $M(\mathcal{A})$ is a closed commutative Banach subalgebra of $B(\mathcal{A})$, the algebra of all bounded linear operators on $\mathcal{A}$, that contains $I$, the identity operator on $\mathcal{A}$, and all multiplication operators. Every multiplier $T$ on a faithful commutative Banach algebra satisfies $T(a b)=a T(b)$ for all $a, b \in \mathcal{A}$. Note that any unital algebra is faithful. Moreover, any semi-prime commutative Banach algebra, i.e. a commutative Banach algebra without non-zero nilpotent elements, is faithful. The reader is referred to [2] for details about multipliers.

Let $X$ be a complex Banach space and let $T \in B(X)$. Then $T$ is an operator with SVEP (single-valued extension property) if, for every open set $U \subseteq \mathbb{C}$, the only analytic function $f: U \rightarrow X$ that satisfies $(T-\lambda) f(\lambda)=0(\lambda \in U)$ is the zero function on $U$ ([3], Definition 1.2.9). We are concerned with the question whether all multipliers on a faithful commutative Banach algebra do have SVEP. The question is stated as an open problem in [3] (Problem 6.2.1). In Section 3 we

Received by the editors March 24, 2006 and, in revised form, June 13, 2006.

2000 Mathematics Subject Classification. Primary 47B40, 47B48.

Key words and phrases. Faithful commutative Banach algebra, multiplier, SVEP.

(C)2007 American Mathematical Society 
shall answer the question in the negative. Before that, in Section 2, we give some sufficient conditions for a multiplier on a faithful commutative Banach algebra to have SVEP.

\section{SUfFicient CONDitions FOR SVEP}

During this section, $\mathcal{A}$ will always mean a faithful commutative Banach algebra and $M(\mathcal{A})$ will be the algebra of all multipliers on $\mathcal{A}$. We shall give some sufficient conditions for $T \in M(\mathcal{A})$ to have SVEP.

The dual space $\mathcal{A}^{*}$ of the algebra $\mathcal{A}$ has a natural structure of a left Banach $\mathcal{A}$-module. Namely, for $a \in \mathcal{A}$ and $\xi \in \mathcal{A}^{*}$, the product $a \cdot \xi \in \mathcal{A}^{*}$ is given by $\langle a \cdot \xi, b\rangle=\langle\xi, a b\rangle$, for all $b \in \mathcal{A}$. For $T \in M(\mathcal{A})$, we shall denote by $T^{*}$ the adjoint operator of $T$ on $\mathcal{A}^{*}$.

Proposition 2.1. If, for a multiplier $T \in M(\mathcal{A})$, the adjoint operator $T^{*} \in B\left(\mathcal{A}^{*}\right)$ has SVEP, then $T$ has SVEP, as well.

Proof. Let $U \subseteq \mathbb{C}$ be an arbitrary non-empty open set and let $f: U \rightarrow \mathcal{A}$ be an analytic function such that $(T-\lambda) f(\lambda)=0$, for all $\lambda \in U$. Choose an arbitrary $\xi \in \mathcal{A}^{*}$ and denote $F_{\xi}(\lambda)=f(\lambda) \cdot \xi$. It is obvious that $F_{\xi}: U \rightarrow \mathcal{A}^{*}$ is an analytic function. Since

$$
\left\langle\left(T^{*}-\lambda\right) F_{\xi}(\lambda), a\right\rangle=\langle\xi, f(\lambda)((T-\lambda) a)\rangle=\langle\xi,((T-\lambda) f(\lambda)) a\rangle=0 \quad(\lambda \in U)
$$

holds for all $a \in \mathcal{A}$ we conclude that $\left(T^{*}-\lambda\right) F_{\xi}(\lambda)=0$, for $\lambda \in U$. By the assumption, $T^{*}$ has SVEP, therefore $F_{\xi} \equiv 0$ on $U$. Thus, $f(\lambda) \cdot \xi=0$, for all $\xi \in \mathcal{A}^{*}$ and all $\lambda \in U$. This gives $\langle\xi, f(\lambda) a\rangle=0$, for all $a \in \mathcal{A}, \xi \in \mathcal{A}^{*}$, and $\lambda \in U$. By the faithfulness of $\mathcal{A}$, we conclude $f \equiv 0$ on $U$.

Let $X$ be a Banach space, $T \in B(X)$, and let $\mathcal{B} \subseteq B(X)$ be a closed subspace such that $T B \in \mathcal{B}$, for any $B \in \mathcal{B}$. It is not hard to see that the multiplication operator $L_{T}$ that is induced by $T$ on $\mathcal{B}$ has SVEP whenever $T$ has SVEP. The following proposition asserts that the converse holds if $\mathcal{X}=\mathcal{A}, T \in M(\mathcal{A})$, and $\mathcal{B}$ contains $L(\mathcal{A})$, the space of all multiplication operators.

Proposition 2.2. Let $T \in M(\mathcal{A})$ and assume that $\mathcal{B} \subseteq B(\mathcal{A})$ is a closed subspace such that $L(\mathcal{A}) \subseteq \mathcal{B}$ and $T B \in \mathcal{B}$, for any $B \in \mathcal{B}$. Then $T$ has SVEP whenever the multiplication operator $L_{T}$ that is induced by $T$ on $\mathcal{B}$ has SVEP.

Proof. If $T$ does not have SVEP, then there exist a non-empty open set $U \subseteq \mathbb{C}$ and a non-zero analytic function $f: U \rightarrow \mathcal{A}$ such that $(T-\lambda) f(\lambda)=0$, for all $\lambda \in U$. Define $F: U \rightarrow \mathcal{B}$ by $F(\lambda)=L_{f(\lambda)}$. Since $\mathcal{A}$ is faithful $F$ is a non-zero analytic function. Of course, $\left(L_{T}-\lambda\right) F(\lambda)=0$, for all $\lambda \in U$, which shows that $L_{T}$ is without SVEP.

Let $T$ be a multiplier on a faithful commutative Banach algebra $\mathcal{A}$. If $\alpha$ and $\beta$ are distinct eigenvalues of $T$ and $a, b \in \mathcal{A}$ are corresponding eigenvectors, then $a b=0$. This follows from $\alpha a b=(T a) b=a(T b)=\beta a b$. Similarly, if $\alpha$ is an eigenvalue of $T, \beta \neq \alpha$ is an eigenvalue of $T^{*}$, and $a \in \mathcal{A}, \xi \in \mathcal{A}^{*}$ are corresponding eigenvectors, then $a \cdot \xi=0$. Namely, it is easily seen that $(T a) \cdot \xi=a \cdot\left(T^{*} \xi\right)$ holds for all $a \in \mathcal{A}$ and $\xi \in \mathcal{A}^{*}$. Thus, the assertion follows from the equality $\alpha a \cdot \xi=(T a) \cdot \xi=a \cdot\left(T^{*} \xi\right)=\beta a \cdot \xi$. Also, a similar assertion holds if $T^{*}$ is replaced by multiplication operator $L_{T}$ on $\mathcal{B}$, where $\mathcal{B} \subseteq B(\mathcal{A})$ is a closed subspace 
that contains $L(\mathcal{A})$ and is invariant for multiplication by $T$ (i.e. $T B \in \mathcal{B}$, for any $B \in \mathcal{B})$.

Proposition 2.3. Let $T \in M(\mathcal{A})$. Let $U, V \subseteq \mathbb{C}$ be open sets and let $f: U \rightarrow \mathcal{A}$ and $g: V \rightarrow \mathcal{A}$ be analytic functions such that $(T-\lambda) f(\lambda)=0(\lambda \in U)$ and $(T-\mu) g(\mu)=0(\mu \in V)$. Then $f^{(m)}(\lambda) g^{(n)}(\mu)=0$, for all $\lambda \in U$ and $\mu \in V$, where $f^{(m)}$ and $g^{(n)}$ are derivatives of $f$ and $g$ of orders $m \geq 0$ and $n \geq 0$, respectively.

Proof. If $\lambda \in U$ and $\mu \in V$ are distinct, then $f(\lambda) g(\mu)=0$, by the observation above. Since $f$ and $g$ are continuous we conclude that $f(\lambda) g(\lambda)=0$ holds as well (for $\lambda \in U \cap V$ ). By double induction, $f^{(m)}(\lambda) g^{(n)}(\mu)=0$.

The following corollary was obtained, with a different proof, as Proposition 4.2.1 in [3].

Corollary 2.4. Any multiplier on a semi-prime commutative Banach algebra has SVEP.

Proof. Let $T \in M(\mathcal{A})$. If $U \subseteq \mathbb{C}$ is a non-empty subset and $f: U \rightarrow \mathcal{A}$ is an analytic function such that $(T-\lambda) f(\lambda)=0$, for all $\lambda \in U$, then $f(\lambda)^{2}=0(\lambda \in U)$, by Proposition 2.3. It follows that $f \equiv 0$ on $U$, by semi-primeness.

Remark 2.5. Let $T$ be a multiplier on $\mathcal{A}$ and let $T^{*} \in B(\mathcal{A})$ be its adjoint operator. If $U, V \subseteq \mathbb{C}$ are non-empty open sets and $f: U \rightarrow \mathcal{A}, G: V \rightarrow \mathcal{A}^{*}$ are analytic functions such that $(T-\lambda) f(\lambda)=0$, for all $\lambda \in U$, and $\left(T^{*}-\mu\right) G(\mu)=0$, for all $\mu \in V$, then $f^{(m)}(\lambda) \cdot G^{(n)}(\mu)=0$, for all $\lambda \in U, \mu \in V$ and all non-negative integers $m$ and $n$. The proof of this assertion is a simple modification of the proof of Proposition 2.3. A similar assertion holds if $T^{*}$ is replaced by $L_{T}$, where $L_{T}$ is the multiplication operator induced by $T$ on $\mathcal{B}$ (a closed subspace of $B(\mathcal{A})$ that contains $L(\mathcal{A})$ and is invariant for multiplication by $T)$.

Let $\mathcal{B} \subseteq \mathcal{A}$ be a subalgebra. We shall say that the multiplication on $\mathcal{B}$ is trivial if $a b=0$, for all $a, b \in \mathcal{B}$.

Proposition 2.6. If each subalgebra $\mathcal{B}$ of $\mathcal{A}$ on which the multiplication is trivial is of finite dimension or of finite co-dimension, then every multiplier on $\mathcal{A}$ has SVEP.

Proof. Assume towards a contradiction that there is a multiplier $T \in M(\mathcal{A})$ without SVEP. Then there exist a non-empty open set $U \subseteq \mathbb{C}$ and a non-zero analytic function $f: U \rightarrow \mathcal{A}$ such that $(T-\lambda) f(\lambda)=0$, for all $\lambda \in U$. There is no loss of generality if we assume that $U=\{\lambda \in \mathbb{C} ;|\lambda|<1\}$. Thus, there exist elements $a_{n} \in \mathcal{A}$, not all zero, such that

$$
f(\lambda)=\sum_{n=0}^{\infty} a_{n} \lambda^{n} \quad(\lambda \in U) .
$$

Of course, we may assume that $a_{0} \neq 0$. Since $T f(\lambda)=\lambda f(\lambda)$, for all $\lambda \in U$, we get, by comparing the coefficients, $T a_{0}=0$ and $T a_{n}=a_{n-1}$, for $n \geq 1$. It follows that

$$
a_{n} a_{m}=\left(T^{m+1} a_{n+m+1}\right) a_{m}=a_{n+m+1}\left(T^{m+1} a_{m}\right)=0,
$$

for all non-negative integers $m$ and $n$. Denote by $\mathcal{B}$ the subalgebra of $\mathcal{A}$ that is generated by $\left\{a_{n} ; n \geq 0\right\}$. It is obvious that the multiplication on $\mathcal{B}$ is trivial. For every $n \geq 0$, the set $\left\{a_{0}, \ldots, a_{n}\right\}$ is linearly independent. Namely, assume that this is not the case. Then there exists a minimal $n \geq 1$ such that $a_{n}=$ 
$\alpha_{0} a_{0}+\cdots+\alpha_{n-1} a_{n-1}$, for some complex numbers $\alpha_{0}, \ldots, \alpha_{n-1}$. However, then we also have $a_{n-1}=T a_{n}=\alpha_{0} T a_{0}+\cdots+\alpha_{n-1} T a_{n-1}=\alpha_{1} a_{0}+\cdots+\alpha_{n-1} a_{n-2}$, which is impossible, by the minimality of $n$. We conclude that $\mathcal{B}$ is not of finite dimension.

Since $f$ is a non-zero analytic function on $U$ there exists $\xi \in \mathcal{A}^{*}$ such that $F(\lambda):=f(\lambda) \cdot \xi$ is a non-zero analytic function on $U$ with values in $\mathcal{A}^{*}$. Of course, $\left(T^{*}-\lambda\right) F(\lambda)=0$, for all $\lambda \in U$. Let $\xi_{n}:=a_{n} \cdot \xi(n \geq 0)$. Then $T^{*} \xi_{n}=\xi_{n-1}$, for $n \geq 1$, and $T^{*} \xi_{0}=0$. A similar argument as before shows that the set $\left\{\xi_{n} ; n \geq k\right\}$ is linearly independent, where $k$ is the smallest non-negative integer such that $\xi_{k} \neq 0$. Since $\left\langle\xi_{n}, b\right\rangle=\left\langle\xi, a_{n} b\right\rangle=0$, for all $b \in \mathcal{B}$ and all $n \geq k$, we conclude that $\mathcal{B}^{\perp}:=\left\{\eta \in \mathcal{A}^{*} ;\langle\eta, b\rangle=0\right.$, for all $\left.b \in \mathcal{B}\right\}$ is not of finite dimension. This is in contradiction with the assumption that $\mathcal{B}$ or $\mathcal{B}^{\perp}$ is of finite dimension.

Remark 2.7. By simple modifications of some proofs in this section it is possible to show that $T \in M(\mathcal{A})$ has SVEP if each subalgebra of $M(\mathcal{A})$ with trivial multiplication is of finite dimension or finite co-dimension. Also, if each submodule of $\mathcal{A}^{*}$ with trivial multiplication has finite dimension or co-dimension, every multiplier on $\mathcal{A}$ has SVEP.

\section{A counterexample}

In this section we shall show that there exist a unital commutative Banach algebra and a multiplication operator without SVEP on it. This answers the question 6.2.1 in 3 in the negative.

Let $\mathcal{A}$ be a Banach algebra (not necessarily commutative) and let $\mathcal{X}$ be a Banach $\mathcal{A}$-bimodule.

Proposition 3.1. The direct sum $\mathcal{A} \oplus X$ is a Banach algebra through the norm and multiplication that are given by

$\|a \oplus x\|_{1}=\|a\|+\|x\|, \quad(a \oplus x)(b \oplus y)=a b \oplus(a \cdot y+x \cdot b) \quad(a \oplus x, b \oplus y \in \mathcal{A} \oplus X)$. If $\mathcal{A}$ has unit, say $e$, then $e \oplus 0$ is unit for $\mathcal{A} \oplus X$.

Proof. Straightforward; see the proof of Theorem 5 in [4].

Corollary 3.2. Let $\mathcal{X}$ be a Banach space and let $\mathcal{A} \subset B(\mathcal{X})$ be a closed commutative subalgebra; then $\mathcal{A} \oplus \mathcal{X}$ is a commutative Banach algebra for the multiplication

$$
(A \oplus x)(B \oplus y)=A B \oplus(A y+B x) \quad(A \oplus x, B \oplus y \in \mathcal{A} \oplus \mathcal{X}) .
$$

If $\mathcal{A}$ contains the identity operator $I$, then $\mathcal{A} \oplus \mathcal{X}$ is unital with unit $I \oplus 0$.

Proof. If $\mathcal{A}$ is a commutative Banach algebra and $\mathcal{X}$ is a left Banach $\mathcal{A}$-module, then $\mathcal{X}$ can be considered as a Banach $\mathcal{A}$-bimodule if the right module structure on $\mathcal{X}$ is given by $x \cdot a:=a \cdot x$.

The following proposition may be deduced from the results in [1]. However, we shall include a more transparent and direct proof of it.

Proposition 3.3. Let $X$ be a Banach space and let $\mathcal{A} \subset B(X)$ be a closed commutative subalgebra that contains $I$. Then, for an operator $S \in \mathcal{A}$, the following assertions are equivalent:

(a) $S$ has SVEP;

(b) for every $x \in X$, the multiplication operator $L_{S \oplus x}$ on $\mathcal{A} \oplus \mathcal{X}$ has SVEP;

(c) there exists $x \in \mathcal{X}$ such that $L_{S \oplus x}$ has SVEP. 
Proof. Assume that there exists $x \in \mathcal{X}$ such that $L_{S \oplus x}$ is without SVEP. Let $U \subseteq \mathbb{C}$ be a non-empty open set and $F: U \rightarrow \mathcal{A} \oplus X$ a non-zero analytic function such that $\left(L_{S \oplus x}-\lambda\right) F(\lambda)=0$, for $\lambda \in U$. Of course, $F(\lambda)=\varphi(\lambda) \oplus f(\lambda)$, for analytic functions $\varphi: U \rightarrow \mathcal{A}$ and $f: U \rightarrow \mathcal{X}$. It follows from $\left(L_{S \oplus x}-\lambda\right) F(\lambda)=0$ that $(S-\lambda) \varphi(\lambda) \oplus(\varphi(\lambda) x+(S-\lambda) f(\lambda))=0$ and therefore

$$
\left(L_{S}-\lambda\right) \varphi(\lambda)=0 \quad \text { and } \quad \varphi(\lambda) x+(S-\lambda) f(\lambda)=0 \quad(\lambda \in U),
$$

where $L_{S}$ is the multiplication operator induced by $S$ on $\mathcal{A}$. If $\varphi$ is non-zero, then $L_{S}$ does not have SVEP and consequently $S$ does not have SVEP. On the other hand, if $\varphi \equiv 0$ on $U$, then $(S-\lambda) f(\lambda)=0$, for $\lambda \in U$, and $f$ is a non-zero function. Thus, again, $S$ does not have SVEP. This proves the implication $(\mathrm{a}) \Rightarrow(\mathrm{b})$. The implication $(\mathrm{b}) \Rightarrow(\mathrm{c})$ is obvious.

Assume that $S \in \mathcal{A}$ does not have SVEP and let $x \in \mathcal{X}$ be an arbitrary vector. Then there exists a non-empty open subset $U \subseteq \mathbb{C}$ and a non-zero analytic function $f: U \rightarrow X$ such that $(S-\lambda) f(\lambda)=0$, for all $\lambda \in U$. Define $F(\lambda)=0 \oplus f(\lambda)$, for $\lambda \in U$. Then $F: U \rightarrow \mathcal{A} \oplus X$ is a non-zero analytic function such that $\left(L_{S \oplus x}-\lambda\right) F(\lambda)=0$, for all $\lambda \in U$. Thus (a) follows from (c).

Let $S$ be an operator without SVEP on a Banach space $\mathcal{X}$ and let $\mathcal{A} \subseteq B(X)$ be the closed subalgebra that is generated by $S$ and $I$. Then $\mathcal{A} \oplus X$ is a faithful commutative Banach algebra such that the multiplication operator $L_{S \oplus 0}$ on $\mathcal{A} \oplus \mathcal{X}$ does not have SVEP, by Proposition 3.3 .

\section{ACKNowledgments}

The authors are grateful to the referee for several helpful remarks and suggestions on a former version of this paper.

\section{REFERENCES}

[1] C. Benhida, E. H. Zerouali and H. Zguitti, Spectra of upper triangular operator matrices, Proc. Amer. Math. Soc. 133(2005), 3013-3020. MR2159780 (2006i:47005)

[2] R. Larsen, An Introduction to the Theory of Multipliers, Springer, 1971. MR0435738 (55:8695)

[3] K. B. Laursen and M. M. Neumann, An Introduction to Local Spectral Theory, Clarendon, Oxford, 2000. MR1747914 (2001k:47002)

[4] V. Müller, Adjoining inverses to noncommutative Banach algebras and extensions of operators, Studia Math. 91(1988), no. 1, 73-77. MR0957286 (90a:46117)

University of Ljubljana, IMFM, Jadranska ul. 19, SI-1000 Ljubluana, Slovenia

E-mail address: janko.bracic@fmf.uni-lj.si

University of Ljubljana, FMF, Jadranska Ul. 19, SI-1000 Ljubljana, Slovenia

E-mail address: martin.jesenko@fmf .uni-lj.si 